\title{
The Time! there is Nothing Simpler than Time
}

\author{
Berov G Lyubomir* \\ Engineer, Independent Innovative Ideas Researcher, Smolyan, Bulgaria \\ *Corresponding author: Berov G Lyubomir, Engineer, Independent Innovative Ideas Researcher, Smolyan 4700, Bulgaria
}

\begin{tabular}{|c|c|}
\hline ARTICLE INFO & ABSTRACT \\
\hline Received: 㓞 July 01, 2021 & $\begin{array}{l}\text { Dear reader, can you imagine for a moment how all fundamental forces act } \\
\text { simultaneously and continuously in our material world? Certainly you can't and no one }\end{array}$ \\
\hline Published: 幽 July 19, 2021 & $\begin{array}{l}\text { can. And can you explain how this material world of ours does not fall apart instantly } \\
\text { from their impact but instead continues to exist for billions of years? Of course, you can. }\end{array}$ \\
\hline
\end{tabular}

there is Nothing Simpler than Time. Biomed J Sci \& Tech Res 37(2)-2021. BJSTR. MS.ID.005983.
Keywords: Time; Dark energy field; Time energy field; Fundamental forces; Intellect

\section{Opinion}

Dear reader, in my article "Time: Philosophy of Science" I have presented my understanding of time. With this article I want to clarify some of the ideas I have discussed there. In particular, I will separate the intelligent energy field of time from the intelligent field of dark energy, assuming that time is a special case, a concrete manifestation, of dark energy. In summary, my hypothesis is that dark energy is the primary energy that gives birth to the entire material world, but the force that then governs this material world is the intelligent energy field of time. And so, time is a dark energy with which we, humans, are, so to speak, in direct contact. But for now, I think the intellectual level of time is a little higher than our human intellect, and so we don't understand it [1].

Dear reader, don't you think it's time for our lack of understanding of time to change? With this article I will try to make the time more understandable. So, let's set the stage by answering the question of which fundamental forces acting in nature are known to the modern science? For now, they are four types of interactions - strong, electromagnetic, weak and gravitational. The strong and weak interaction are the nuclear interactions. The protons and neutrons that make up the nucleus of an atom are essentially made up of quarks connected to each other by a strong nuclear interaction. The particle carrying this force is gluon. The weak nuclear interaction causes the nuclei of some elements to decay. This force controls a process called "beta decay," in which a neutron spontaneously decays into a proton, electron, and antineutrino [2]
Next in strength is the electromagnetic interaction carried by the photon. It is about 100 times weaker than the strong, but affects unlimited distances, while the strong and the weak act only at the scale of the atom. Finally, there is the gravitational interaction, which is billions upon billions of times weaker than the electromagnetic one and is carried by the graviton. Graviton has not yet been confirmed experimentally. All other forces we observe or know of are the result of these four interactions. For example, friction is a manifestation of an electromagnetic force acting between atoms on two surfaces and Pauli's principle of exclusion, which prevents atoms from passing through each other. The forces in springs modeled by Hooke's law are the result of electromagnetic force and Pauli's principle, which join actions to return the object to its equilibrium position. The centrifugal force is fictitious and is apparent only in a rotating reference frame. And so on and so forth.

Dear reader, can you imagine for a moment how all fundamental forces act simultaneously and continuously in our material world? Certainly you can't and no one can. And can you explain how this material world of ours does not fall apart instantly from their impact, but instead continues to exist for billions of years? Of course, you can. We humans, when confronted with indisputable facts, always consider many different possible answers, and, after long research we sometimes come to the almost correct one. It is worth taking the necessary time and resources to solve the above mystery. In my hypothesis, one of the possible answers is: there is another force that constantly controls and coordinates the actions 
of all known and unknown forces, and its main goal is the stability of our world in the process of its development and improvement [3].

However, this force must have completely new and unthinkable for now characteristics - it must have unimaginably powerful intelligence, capabilities and durability. Intelligence because only an intelligent creature can have a goal. Capabilities because it must cover the entire universe. Durability because it needs to last for as long as our universe continues to exists. And most importantly, the control should be such that everything happens at a specific time. And so, I think, this force is obviously the time. The notion that has been accepted so far by most scientists is that the time is a sequence of events, or, in other words, a passive "observer". This notion is meaningless and unacceptable to me. I intuitively feel that it is an energy field, a force that has the above characteristics. Another necessary characteristic of this force is that in order to be the most efficient in achieving its goals, it must be spread everywhere in the material world, or as I have already mentioned, it must be an energy field [4].

And how does time interact with the four fundamental forces? My hypothesis requires that these forces, as a prerequisite, also have

ISSN: 2574-1241

DOI: 10.26717/BJSTR.2021.37.005983

Berov G Lyubomir. Biomed J Sci \& Tech Res

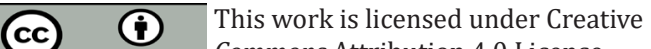
(c) Commons Attribution 4.0 License Submission Link: https://biomedres.us/submit-manuscript.php some beginnings of intellect? Only then could they communicate and be controlled by the aforementioned powerful intellect of time seamlessly and efficiently. In fact, the material objects that in turn are controlled by the fundamental forces must also have some beginnings of intellect. And here I am, struck by a "genius" idea: We, humans, have claim that we have intelligence. But to be controlled? This is unacceptable!!

Dear reader, perhaps it is time to accept the assumption that there are more powerful intellects than ours. Only under this condition the hypothesis presented here can become possible and the world we live in can become understandable and predictable.

\section{References}

1. Berov Lyubomir (2020) A Time: Philosophy of Science. Journal of Research Notes 3(1): 1019.

2. Rubin Vera (1997) Bright Galaxies, Dark Matter. American Journal of Physics 65(10): 1028.

3. Castaneda Carlos (1993) The Art of Dreaming.

4. Stephen W Hawking (1973) A Brief History of Time.

$\begin{array}{ll}\text { BIOMEDICAL } & \text { Assets of Publishing with us } \\ \text { RESEARCHES } & \text { - Global archiving of articles } \\ \text { - Immediate, unrestricted online access }\end{array}$

